\title{
Penetrating the Geometric Module: Catalyzing Children's Use of Landmarks
}

\author{
Alexandra Twyman, Alinda Friedman, and Marcia L. Spetch \\ University of Alberta
}

\begin{abstract}
We used a reference memory paradigm to examine whether 4- and 5-year-old children could be trained to use landmark features to relocate targets after disorientation. In Experiment 1, half of the children were pretrained in a small equilateral triangle-shaped room. Each of the three walls was a different color, and the target was always in the middle of the yellow wall. These children and a control group were tested in a small rectangular room with three white walls and one yellow wall; the target was placed in one of the corners. Children with pretraining responded more frequently to the correct corner than to the diagonally congruent corner on their first set of four trials in the rectangular room, whereas the children in the control group used geometric cues exclusively. Three additional groups of children (Experiment 2) showed that the use of landmark features — both salient and subtle — can be learned in as few as four practice trials in a small rectangular room. The data support the view that both geometry and landmark features are adaptively combined in the same representation.
\end{abstract}

Keywords: children, landmark features, learning, adaptive combination, geometric module

The ability to orient oneself in space is a fundamental skill that has evolutionary and ecological significance for all mobile organisms. Virtually all species tested encode geometric properties of the environment (see K. Cheng \& Newcombe, 2005, for review). Whereas children under 6 years of age (Hermer-Vazquez, Moffet, \& Munkholm, 2001; Hermer \& Spelke, 1996) appear to use geometric cues exclusively, many species are able to conjoin geometric and feature cues, including pigeons (Kelly, Spetch, \& Heth, 1998), rhesus monkeys (Gouteux, Thinus-Blanc, \& Vauclair, 2001), chicks (Vallortigara, Zanforlin, \& Pasti, 1990), and fish (Sovrano, Bisazza, \& Vallortigara, 2002).

K. Cheng (1986; see also K. Cheng \& Gallistel, 1984) first proposed the existence of an encapsulated geometric module devoted to the task of orienting (and reorienting) in space; he hypothesized that the existence of such a module could explain why disoriented rats systematically chose geometrically equivalent corners in a rectangular space when they were trying to relocate a food source they had previously visited. Notably, in a rectangular environment, geometrically equivalent corners are equivalent in both metric (a long wall meets a short wall) and sense (left-right

Alexandra Twyman, Alinda Friedman, and Marcia L. Spetch, Department of Psychology, University of Alberta, Edmonton, Alberta, Canada.

This research was supported by Natural Sciences and Engineering Research Council of Canada Grants to Alinda Friedman and Marcia L. Spetch. We thank Mark Holden, Isaac Lank, John Twyman, and David Delancey for help in constructing the small rooms and Kirsten Williams and Bowie Lui for help in soliciting the participants and scoring the data. We also thank the parents and children who took the time and effort to participate in this study.

Correspondence concerning this article should be addressed to Alexandra Twyman, who is now at the Department of Psychology, Temple University, Weiss Hall, 1701 North 13th Street, Philadelphia, PA 19122. E-mail: atwyman@temple.edu relations) information, so this finding indicates that rats use both types. Cheng's most surprising finding was that in a working memory task in which the correct location changed on each trial, rats used geometric information exclusively even when the corners of the space were completely disambiguated by nongeometric landmarks. In contrast, in a reference memory task in which the correct location remained the same on each trial, the rats learned to use featural information to locate the target in fewer than 30 trials. However, when the featural information was removed from the target corner and the geometrically equivalent corner, the rats were unable to use the remaining cues at the more distant corners and reverted to using geometric cues exclusively. Cheng hypothesized that rats reoriented by using representations encoded in a geometric module, which essentially records metric information about the shape of the environment. Information about landmarks near the target was hypothesized to be "glued" onto the metric frame provided by the geometric module. Subsequent researchers have assumed that such nongeometric features are encoded and represented in separate modules (see K. Cheng \& Newcombe, 2005, for review)

Hermer and Spelke (1994, 1996) extended K. Cheng's (1986) work to human children and found that younger children (between 2 and 4 years of age) behaved identically to the rats. They split their choices evenly between the correct corner and the diagonally opposite corner of a small $4 \times 6 \mathrm{ft}(1.22 \times 1.83 \mathrm{~m})$ rectangular room and did not take advantage of a blue wall-a nongeometric landmark - to relocate an object hidden in one of four corners. In a subsequent study, Wang, Hermer, and Spelke (1999) tested children between 18 and 24 months of age in either a 2-m square room with no landmarks, a square room with a red wall, or a square room with a "bump" in the middle of one of the walls, which they argued was a geometric cue because the wall was still a continuous surface. The children's responses were random in the 
first two cases, in that they were divided equally amongst all four corners, but they picked the correct corner $58 \%$ of the time in the room with the bump (see also Gouteux and Spelke, 2001). The data from Hermer and Spelke $(1994,1996)$ and from Gouteux and Spelke (2001), as well as their own, led Hermer-Vazquez et al. (2001) to propose a model of how young children reorient. Their model suggests that until children have spatial language (roughly around 6 years of age), they will reorient themselves exclusively on the basis of spatial representations within the geometric module and that these representations are common to humans and other mammals.

Recently, Learmonth, Nadel, and Newcombe (2002) challenged the modular view by examining the performance of 5-year-old children in a within-subject design. Half of the children were tested for four trials in a small $4 \times 6 \mathrm{ft}(1.22 \times 1.83 \mathrm{~m})$ room followed by four trials in a large $8 \times 12 \mathrm{ft}(2.44 \times 3.66 \mathrm{~m})$ room, and the remaining children were tested in the reverse order. Both large and small rooms had a blue landmark wall. In contrast to Hermer and Spelke (1996), when tested in the large room the children used the landmark to respond to the correct corner on $57 \%$ of the trials and to the diagonally congruent corner on only $23 \%$ of the trials. However, when tested in the small room, the children split their responses equally between the correct corner and the geometrically congruent corner, thus replicating Hermer and Spelke's finding. Moreover, children were unable to use the landmark in the small room even if they had first been tested in the larger room and were able to use the landmark there. Similarly, Learmonth, Newcombe, and Huttenlocher (2001) found that children as young as 17 to 24 months of age chose the correct corner $60 \%$ of the time in a large rectangular room when the landmark features were a bookcase and a door.

Learmonth et al. (2002) concluded that at least three possibilities could explain the difference between their results and Hermer and Spelke's (1994, 1996). First, perhaps only children older than about 5 years of age, who have more navigational experience, have learned that color is a useful cue no matter what the situation (e.g., a large or small space). If so, then younger children may have failed to generalize what they know about navigation in large spaces to the small experimental spaces used by Hermer and Spelke (1994, 1996). A second possibility is that the demands of the two spaces were somehow different, in that the children might not have perceived the smaller space as one in which navigation was relevant and so ignored the feature information. A related third possibility is that the children were used to navigating in larger spaces and encoded the blue wall in the large room as a heading cue. In contrast, the small room may not have been perceived as a space in which heading information was relevant. In any case, it is clear that under some circumstances, 5-year-olds can use landmarks to reorient themselves, but their use of landmark information is "fragile" (Learmonth et al., 2002). Indeed, in their extensive review of this literature, K. Cheng and Newcombe (2005) concluded that for children, the "size of the space in which they were tested was a key determinant of whether they used the featural properties" (p. 14) to locate the correct corner after disorientation. The fragility of children's landmark use and its variability with room size argues against an encapsulated representation of geometric information. That is, it is difficult to understand how a geometric module that is impenetrable to other information could be active under one circumstance but not another.

The main goal of the present study was to challenge the modularity hypothesis further by examining whether 4- and 5-year-olds can learn to use landmark features in a small space in a relatively short time. If children fail to use landmark features in small enclosures because they simply cannot generalize from their prior navigational experiences in large spaces, then it might be possible to create an experience that would facilitate the generalization and make their use of landmarks less fragile. Further, if children can generalize after a relatively small amount of pretraining, the existence of an encapsulated geometric module would be called into question because, presumably, the influence of such a module should not be overcome so quickly. In addition, if 4-year-olds can learn to use landmark features in a reorientation task, it cannot be that spatial language is necessary for this behavior to emerge (Hermer \& Spelke, 1996; Hermer-Vazquez et al., 2001; see Newcombe \& Ratliff, 2007, for review). However, if there is something about a small space that acts as a general trigger for the children to either ignore or otherwise be unable to use their navigation skills, then pretraining should not help children to overcome their tendency to use exclusively geometric aspects of the environment. Indeed, in Learmonth et al. (2002), even the children who received specific practice with the landmark feature (blue wall) in the larger room first were unable to generalize to the smaller space. Moreover, Wall, Botly, Black, \& Shettleworth (2004) demonstrated that rats that had learned to use a black panel to locate food in a square arena started making rotational errors when transferred to a rectangular arena with the black panel; this was taken as strong evidence for the obligatory nature of geometric cues.

In Experiment 1, 4- and 5-year-old children were tested in a reference memory paradigm in two small rooms. All children were tested for eight trials in a $4 \times 6 \mathrm{ft}(1.22 \times 1.83 \mathrm{~m})$ rectangular room; for the first four trials one of the walls was yellow, and the correct corner was one of the two that were adjacent to the yellow wall. For the second four trials all four walls were white, and the correct corner remained the same for a given child. The main manipulation was that half of the children received pretraining in a room that was an equilateral triangle with an area approximately equal to that of the rectangular room. An equilateral triangle has no unique geometric cues, so geometry per se cannot be used to solve the problem. However, we covered each wall of the triangular room in a different colored fabric (red, yellow, and blue) to provide unique landmarks. Furthermore, the correct choice was always placed in the center of the yellow wall. If the children pretrained in the triangular room learn to pay attention to the yellow wall and use it to solve the problem of locating the target, then when transferred to the rectangular room with the yellow wall, they should choose the correct corner more often than the geometrically congruent corner, and more often than the group that was not pretrained.

Note that if children can learn to pay attention to and encode landmark features, then the triangular room should help in two ways. First, the pretraining emphasizes the importance of paying attention to color in general, and second, it highlights the particular color that signals the correct response (yellow). In addition, because the target is in the middle of the wall, the children do not have to remember a left-right sense relation. This aspect of the 
procedure is crucial for ensuring that pretraining in the triangular room does not merely teach children the correct answer directly (i.e., the yellow wall). Indeed, if that were all the children learned, they could not transfer immediately to the correct corner of the rectangular room because they would still need to learn the left-right sense relations between the walls. Thus, if the children are learning only the correct color, they should respond to both yellow corners in the rectangular room equally often. Similarly, if geometry is compulsive, as with Wall et al.'s (2004) rats, then children should revert to geometry when they are transferred from the triangular room to the rectangular layout. Alternatively, if the pretraining "reminds" children that color can be used as a heading cue or as a means of reorienting, then this should transfer to the rectangular room immediately. Furthermore, when the children in the pretraining group are switched to the all white room, they should revert to responding on the basis of geometry. To foreshadow, our data argue against the idea that geometric information is encapsulated and in favor of the alternative: That human children as young as 4 years can readily use both geometry and features, if available in the environment, to reorient themselves in an object relocation task.

\section{Experiment 1}

\section{Method}

Participants and design. Participants were recruited from daycare centers and YMCA facilities. Information pertaining to ethnicity, socioeconomic status, or the parents' education and occupation was not collected. Parents were given an honorarium. Equal numbers of 4- and 5-year old girls and boys were randomly assigned to either the untrained group, whose activity began immediately in the rectangular room, or to the triangle pretraining condition. Of the 29 children who participated, the data from 5 were discarded because they either did not comply with the procedure or did not want to continue playing the game. The remaining 24 participants had an average age of 58 months. There were twelve 4-year-olds (average age $=52$ months; range $=49-59$ months) and twelve 5-year-olds (average age $=65$ months; range $=61-71$ months).

Half of the children searched in the rectangular room exclusively, and the other half started in the triangular room before advancing to the rectangular room. The criterion for mastering the triangular room was three correct choices in a row. All of the children reached the criterion $(M=6.2$ trials; range $=3-12$ trials $)$. All children participated for eight trials in the rectangular room: four trials with the yellow feature wall and then four trials with all white walls. The target corner remained the same for all eight trials for a given child and was counterbalanced across children within groups.

Apparatus. The triangular and rectangular rooms were located in adjacent $8 \mathrm{ft} 2 \mathrm{in} . \times 7 \mathrm{ft} 3$ in. $(2.49 \times 2.21 \mathrm{~m})$ laboratory rooms. An overhead camera was mounted in the ceiling of the rectangle room. There was one point of entry per search space. Both rooms were constructed out of $5 \mathrm{ft} 11 \mathrm{in}$. $(1.80 \mathrm{~m})$ tall office dividers held together with a wooden frame and exterior brackets. The base plates of the dividers were removed so that the bottoms of the dividers were flush with the floor. Vapor barrier sheets were sandwiched between the divider and the top wooden frame so that light could penetrate the area but no external cues could be seen. The dividers were covered with cotton fabric on their interior sides.

The equilateral triangle had walls that were $7 \mathrm{ft} 4$ in. $(2.24 \mathrm{~m})$ long, creating a 23-square-foot (2.1-square-meter) search space. Each wall was completely covered with red, yellow, or blue fabric. The entrance was between the blue and yellow walls. A flap of fabric $3 \mathrm{ft} 11 \mathrm{in}$. (1.19 $\mathrm{m})$ high from the floor was not stapled to the wall and could be pulled outwards to allow the experimenter and child to enter the room. Velcro allowed the fabric to be pulled closed and fastened, leaving a smooth surface.

The walls of the rectangular room were $5 \mathrm{ft} 11 \mathrm{in} .(1.80 \mathrm{~m})$ long and $4 \mathrm{ft}(1.22 \mathrm{~m})$ wide, creating a search space of 23.7 square feet (2.2 square meters). Three of the walls (two long and one short) were covered with off-white fabric. The remaining short wall was covered with either off-white or yellow fabric hung from a rectangular plank. The door was again a fabric flap located between two white walls and was also pulled shut and fastened with Velcro after the experimenter and child had entered the room.

The search stimuli were 8 -in. $(20-\mathrm{cm})$ white squares of laminated paper placed on the floor in both rooms. All of the squares looked identical from the back. On the front of one of the cards in each room was a picture of one of five characters from the movie Madagascar (T. Cheng, Soria, \& Darnell, 2005), including Gloria the hippopotamus, Alex the lion, Marty the zebra, Skipper the penguin, and Melman the giraffe. The cards were placed in the middle of the walls of the triangular room and in the corners of the rectangular room.

Procedure. Parents brought their children into the university for the study. Consent was obtained from the parents, and then the children were given time to become comfortable with their surroundings as well as with the experimenter. Most children were ready to participate at this point. A few of the children were more timid, and they were accompanied by their parents for a tour of the search space before beginning the trials. It was emphasized at the start and after the first trial that the child could stop participating at any time.

Once in the room, the children were shown the cards and told that one of them had an animal on the back. The cover story for the children was based on the movie Madagascar. In essence, the children were told that one of the animals had escaped from the zoo and that it was their task to try and find the animal. A special fort could help them find the animals.

The method of disorientation followed that used by Hermer and Spelke (1994, 1996). For each trial in both rooms, the experimenter turned over the card that had the character on the other side, and the children were asked to remember where the character was hiding. Then they were asked to close their eyes, spin in a circle, and count their footsteps out loud. While the child was rotating, the experimenter walked around the room in the direction opposite to that in which the child was spinning. After the child had rotated at least four times, the experimenter stopped, using the middle of a different wall to stop at for each trial. The child was then asked to stop spinning, at a different wall for each trial, and then to try and find the character. The child was only allowed to make one choice. 
If the choice was incorrect, the experimenter showed the child the correct location by flipping over the correct card. Each child saw each of the five characters at some point in the study to keep his or her interest.

When the children in the pretraining group had reached the criterion of three correct trials in a row in the triangular room, they were told that they were "getting so good at finding the animals that we are going to go to a new fort." Then they were taken to the rectangular room. After the first four trials for both groups, the child was led out of the room by the experimenter, and the yellow wall was changed to a white wall. The children were asked to wait with their parents until the change was completed.

\section{Results}

Figures $1 \mathrm{a}$ and $1 \mathrm{~b}$ show the proportion of responses made to each corner for the group that received pretraining in the triangular room and the group that started in the rectangular room, respectively. The participants who pretrained in the triangular room used the yellow wall successfully as a landmark feature from the beginning of their transfer trials, whereas the unpretrained group used geometric cues exclusively during the initial four trials. Planned comparisons showed that for the first set of four trials the pretrained group went to the correct corner $71 \%(S E M=9 \%)$ of the time and to the diagonally opposite corner $16 \%(S E M=8 \%)$ of the time; $F(1,11)=10.75, M S E=$ $3,276.52, p<.01, \eta_{p}^{2}=.494$. In contrast, during the second four trials, which had all white walls, they went to these corners $38 \%(S E M=6 \%)$ and $35 \%(S E M=7 \%)$ of the time, respectively, $F(1,11)<1.00$. The group that received no pretraining went to the correct corner $44 \%(S E M=8 \%)$ of the time and to the diagonally opposite corner $33 \%(S E M=7 \%)$ of the time, $F(1,11)<1.00$, during their first four trials with the yellow wall. Curiously, during the second set of trials, when all of the walls were white, this control group went to the correct corner $56 \%(S E M=9 \%)$ of the time and to the congruent corner $15 \%$ $(S E M=6 \%)$ of the time, $F(1,11)=10.58, M S E=1,969.70$, $p<.01, \eta_{p}^{2}=.490$.

An analysis of variance (ANOVA) with group (pretraining vs. no pretraining) and age (4 and 5 years) as the between-subjects factors and corner (correct vs. diagonally opposite) and half (first vs. second four trials) as within-subject factors yielded the expected group by corner by half interaction, $F(1,20)=14.98, M S E=695.31, p<.01$, $\eta_{p}^{2}=.428$. In addition, there were main effects of corner, $F(1,20)=$ 12.64, $M S E=1,393.23, p<.01, \eta_{p}^{2}=.387$, and age, $F(1,20)=$ 4.74, $M S E=351.56, p=.04, \eta_{p}^{2}=.192$. Across the control and pretraining groups the 4-year-olds showed a greater tendency to choose the correct corner over the diagonally opposite corner than did the 5-year-olds during both the first and second sets of four trials: For the 4-year-olds the difference between corners was $42 \%\left(S E_{\text {diff }}=\right.$ $16 \%)$ on the first set of trials and $29 \%\left(S E_{\text {diff }}=12 \%\right)$ on the second set, and for the 5-year-olds the differences were $23 \%\left(S E_{\text {diff }}=15 \%\right)$ and $15 \%\left(S E_{\text {diff }}=14 \%\right)$, respectively. No effects interacted with age, and no other effects were significant.

\section{Experiment 2}

The first experiment provided evidence that a relatively small amount of pretraining in an enclosure where it was not possible to

\section{$1^{\text {st }}$ Four Trials $\quad 2^{\text {nd }}$ Four Trials}

a. Triangle Pretraining: 4 Trials Yellow Wall -4 Trials White Wall

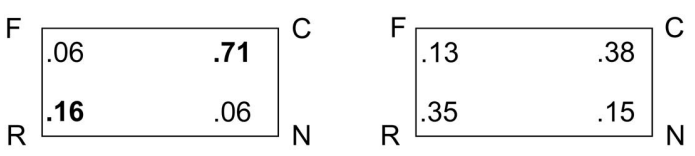

b. No Pretraining: 4 Trials Yellow Wall - 4 Trials White Wall (Gap)

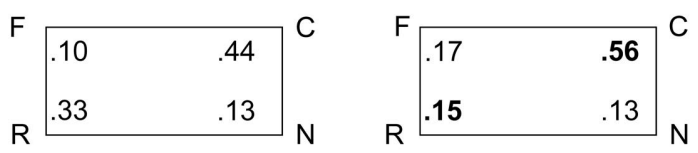

c. No Pretraining: 8 Trials - Yellow Wall

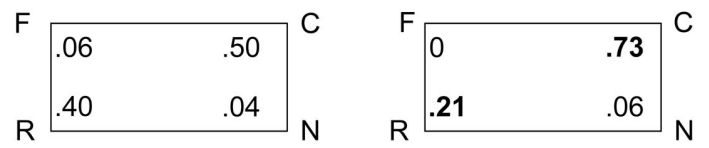

d. No Pretraining: 8 Trials - White Wall (Gap)

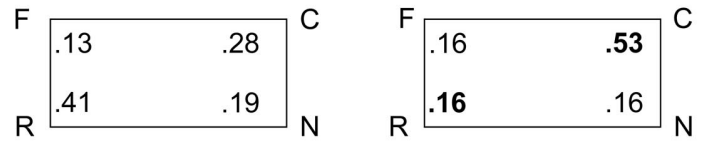

e. No Pretrainina: 8 Trials - White Wall
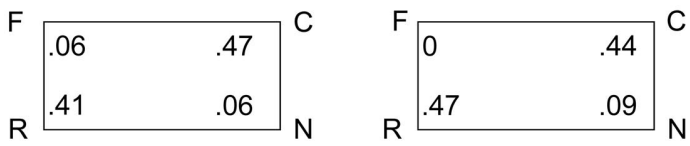

Figure 1. The proportion of participants' responses for each of five groups to each of four corners of a $4 \times 6 \mathrm{ft}(1.22 \times 1.83 \mathrm{~m})$ rectangular room when they were relocating a hidden target after having been disoriented. The responses were aggregated separately for the first four trials and the second four trials. $\mathrm{C}=$ the correct corner; $\mathrm{R}=$ a rotational error made to the corner that is geometrically congruent to the correct corner; $\mathrm{N}=$ an error made to the corner that is nearest to the correct corner; $\mathrm{F}=$ an error made to the corner that is far from the correct corner but is not geometrically congruent to it. The numbers in bold within a given rectangle indicate a significant difference between the correct and geometrically congruent corners and are evidence of landmark use. Groups a and b are from Experiment 1, and groups c, d, and e are from Experiment 2.

use geometric cues facilitated the immediate use of features during a transfer test. However, there was one aspect of the procedure and one aspect of the data that warranted caution in interpreting these results. From the procedural standpoint, the pretrained group received more trials overall than the untrained group (from as few as 3 to as many as 12 pretraining trials). Consequently, the fact that children in the untrained group did not use the yellow wall during their first four trials in the rectangular enclosure might reflect a general lack of practice rather than a lack of specific pretraining with the feature wall. This is not as implausible as it seems; to our 
knowledge, all previous studies with young children have used at most four trials in a given room. ${ }^{1}$

To examine the possibility that practice alone might facilitate the use of features to reorient, we tested a group of children in the rectangular room with the yellow wall present for all eight trials and analyzed the data separately for the first and second sets of four trials. We will refer to this group as the "yellow-wall" group. We anticipated that if merely practicing with a landmark feature for four trials enables young children to be able to use it to respond correctly in a small room, then the data for the second set of four trials in the yellow-wall group should replicate the data from the first set of four trials for the group trained in the triangular room in Experiment 1. Further, if such a small amount of practice enables children to use landmark information, then it is difficult to argue that the geometric module is obligatory or even necessary to explain their reorienting behavior.

The second issue was raised by the data themselves. The children in the untrained group of Experiment 1 appeared to use some sort of feature during the second set of four trials when, ostensibly, there should have been none. Inspection of the rectangular room showed that the fabric on the removable white wall that replaced the removable yellow wall had a gap of approximately 1.5 in. (4 $\mathrm{cm}$ ) between the bottom of the fabric and the floor, leaving the grey fabric on the room divider visible; the fabric on the other white walls and the yellow wall all abutted the floor. It was possible that this gap in the white wall was treated as a feature. If so, the children in the untrained group may have started to learn to use the yellow wall as a landmark feature by the end of the first four trials and then, when the yellow wall was switched to the white wall with the gap, noticed the gap and were able to use it as a basis for responding. The triangle pretrained group, on the other hand, may have been overtrained with the yellow wall (i.e., they had both the pretraining trials in the triangular room and the four experimental trials in the rectangular room), and this overtraining may have prevented them from immediately noticing the gap in the white wall during their second set of four trials in the rectangular room.

To test this idea, we ran trials with two additional groups of children. For the "white-wall-gap" group we tested the children for eight trials in the original rectangular room with the white wall that contained the 1.5-in. (4-cm) gap between the fabric and the floor. If they were able to use this gap as a feature, then they should respond to the correct corner more frequently than to the geometrically congruent corner during the second set of four trials and thus replicate the data for the second set of trials in the original untrained group (Figure 1b).

For the "white-wall" group we constructed a new rectangular room that was identical in size to the original, except that all four white walls were covered with fabric that abutted the floor. Thus, there was no unique feature that could be used to respond, and we expected that this group would use geometry exclusively during both the first and second sets of four trials.

\section{Method}

Participants. For the yellow-wall group, 7 girls and 6 boys participated. The data from 1 girl were discarded because she did not count or spin. The remaining 12 participants were eight 4-year- olds (average age $=54$ months; range $=51-58$ months) and four 5-year-olds (average age $=66$ months; range $=61-70$ months). Information pertaining to ethnicity, socioeconomic status, or the parent's education and occupation was not collected.

There were 3 girls and 5 boys in the white-wall-gap group. No child's data were discarded. The average age of the three 4-yearolds was 54 months (range $=50-59$ months), and the average age of the five 5-year-olds was 64 months (range $=61-72$ months).

There were 4 girls and 4 boys in the white-wall group. No child's data were discarded. The average age of the three 4-yearolds was 52 months (range $=49-54$ months), and the average age of the five 5-year-olds was 66 months (range $=61-70$ months).

Apparatus and procedure. The apparatus for the yellow-wall group and the white-wall-gap group was the same as the rectangular search space in Experiment 1. The apparatus for the new white-wall group was identical in size to the original rectangular room but was built out of $2 \times 2$ in. $(5 \times 5 \mathrm{~cm})$ framing. One of the short walls was hinged so that it could function as a door. The fabric covering the walls was stapled in such a way that once the door was closed the walls were indistinguishable from each other except for their lengths (long and short).

The procedure for all three groups of participants was identical to the untrained condition of the first experiment. This included a break during which the children left the rectangular room for $3 \mathrm{~min}$ between the first four and the last four trials even though nothing changed in the room. The correct corner was again the same for all eight trials for a given child and counterbalanced across children within a group.

\section{Results}

Figures $1 \mathrm{c}, 1 \mathrm{~d}$, and 1e show the data for the yellow-wall group, the white-wall-gap group, and the white-wall group, respectively. The pattern of data is exactly as anticipated if participants could learn to attend to a feature by the end of the first four trials in the yellow-wall and white-wall-gap groups but not in the white-wall group. Planned contrasts showed that for the yellow-wall group, there was no difference in proportion of responses to the correct or diagonally opposite corner on the first set of four trials $(50 \%$ $[S E M=9 \%]$ vs. $40 \%[S E M=10 \%]$, respectively), $F(1,11)<$ 1.00 , but there was a large effect of corner during the second set of

\footnotetext{
${ }^{1}$ Using a reference memory task, Hermer-Vazquez, Moffet, \& Munkholm (2001) gave children three to four trials in each of two conditions in the same small rectangular room with a blue landmark wall. In the direct landmark condition, a toy was hidden in the middle of one of the walls and in the indirect landmark condition, it was hidden in a corner. The conditions were presented in counterbalanced order. Both 3- to 4-year-olds and 5- to 6-year-olds searched successfully at the correct wall in the direct landmark condition, but only the older children searched the correct corner significantly more often that the diagonally congruent corner in the indirect landmark condition. However, the authors do not report the data separately by presentation order, and the younger children's data in the indirect landmark condition were trending towards a higher percentage of responding to the correct corner. It may be that the 3- to 4-year-old children who received the direct landmark condition first did use the feature information more often than the geometric information to respond when they were transferred to the indirect landmark condition, and the lack of statistical significance reflects averaging over presentation order.
} 
four trials $(73 \%[S E M=8 \%]$ vs. $21 \%[S E M=7 \%]$, respectively), $F(1,11)=12.21, M S E=2,665.72, p<.01, \eta_{p}^{2}=.526$. In fact, the data for the second set of four trials in the yellow-wall group were nearly identical to the data for the first set of trials in that room for children in the triangular pretraining group of Experiment 1. Further, an ANOVA with age as the between-subjects factor and corner and half as the within-subject factors for the yellow-wall group of Experiment 2 showed no main effect or interactions with age, but the expected half by corner interaction approached significance, $F(1,10)=4.41, M S E=1,156.25, p<.07, \eta_{p}^{2}=.306$.

Similar to the results for the yellow-wall group, for the whitewall-gap group there was no difference in proportion of responses to the correct or diagonally opposite corner on the first set of four trials $(28 \%[S E M=7 \%]$ vs. $41 \%[S E M=7 \%]$, respectively), $F(1$, $7)=1.17, p=.32, \eta_{p}^{2}=.143$, but there was a difference on the second set of trials $(53 \%[S E M=10 \%]$ vs. $16 \%[S E M=5 \%]$, respectively), $F(1,7)=9.00, M S E=1,250.00, p<.02, \eta_{p}^{2}=$ .563. The data on the second set of trials for this group nicely replicated the data for the second set of trials for the untrained group of Experiment 1 (Figure 1b), as predicted. The ANOVA for the white-wall-gap group with age as a factor yielded only the expected half by corner interaction, $F(1,6)=8.95, M S E=$ 559.03, $p<.02, \eta_{p}^{2}=.599$.

In contrast to the data for the children in the yellow-wall and white-wall-gap groups, both of which showed evidence of having learned to use quite different types of features in a fairly short time period, the children in the "true" white-wall group responded solely on the basis of geometry for both the first and second set of trials, $F(1,7)<1.00$ in both cases (Figure 1e).

\section{Discussion}

Four out of five groups of young children rapidly learned to use landmarks to relocate a hidden object in a small room after having been disoriented. One group was pretrained to find the object in the middle of a yellow wall of a small room shaped like an equilateral triangle and then transferred to a small rectangular room in which the landmark was still a yellow wall, but the object was in a corner. The remaining three groups received no specific training outside of the environment in which they were to perform, yet each was able to use landmark information-either the yellow wall or a one and a half inch gap between the floor and the fabric of a white wall- to respond to the correct corner of the rectangular room after only four practice trials. The only group that did not use landmark information was the group that had none to use.

In some ways, the most interesting data are from the untrained group in Experiment 1. The children in this group received four trials in the small rectangular room with the yellow wall as a landmark and then four trials in the same room with the yellow wall having been changed to a white wall that, inadvertently, had a gap between the bottom of its white fabric and the floor. Eight of the 12 children responded more frequently to the correct corner than to the diagonally opposite corner during the second set of four trials (for these children the percentages of responses to the correct and congruent corners were $68.8 \%$ and $3.1 \%$, respectively, and the percentages for the group as a whole were $56 \%$ and $15 \%$, respectively). Thus, not only were these children able to use landmarks after four practice trials, they generalized what they had learned during the first four trials to a completely new type of landmark cue which, arguably, was a much more subtle one. The similarity between the data from the second set of four trials in both untrained group in Experiment 1 and the white-wall-gap group in Experiment 2 is striking. Both groups of children used the 1.5-in. $(3.8-\mathrm{cm})$ gap as a landmark and chose the correct corner more often than the congruent corner with similar frequencies: The percentages were $56 \%$ and $15 \%$, respectively, for children from Experiment 1 and $53 \%$ and $16 \%$, respectively, for children from Experiment 2.

Equally striking is the similarity between the data from the group that received pretraining in the triangular room of Experiment 1 and the group from Experiment 2 that started out in the rectangular room immediately and had eight trials with the yellow wall present. The data for the first four trials of the pretrained group when it was moved to the rectangular room were nearly identical to the data for the second four trials of the untrained group that started out in the rectangular room: Nine of the 12 children in the pretrained group of Experiment 1 and 8 of the 12 children in the untrained yellow-wall group of Experiment 2 responded significantly more to the correct corner $(86.1 \%$ and $87.5 \%$, respectively) than to the geometrically opposite corner ( $3.1 \%$ vs. $6.3 \%$, respectively). For children pretrained in the triangular room this transfer performance implies that they not only learned that color was important, but that they were able to make immediate use of sense (left-right) information to find the correct corner when they were transferred to the rectangular room. Notably, therefore, these children could not have been using the yellow wall merely as a beacon. For the children who started out in the rectangular room in Experiment 2, it took a mere four trials for them to achieve this level of performance. Further, all of the children were young enough to preclude spatial language having played a major role in their success.

Given how readily Experiment 1's untrained group and Experiment 2's gap group learned to use the gap as a landmark, it may appear odd that the group pretrained in the triangular room and that so successfully and immediately transferred to using the yellow wall as a landmark in the rectangular room reverted to geometry when the yellow wall was changed to the white wall with the gap. However, the group pretrained in the triangular room had an average of 6 pretraining trials (range $=3-12$ ) in which the yellow wall cued the correct response and then four more trials in the rectangular room with the yellow wall. Assuming that color is a reasonably salient landmark, it may be that color overshadowed the more subtle gap cue after the yellow wall was removed. In addition, the children pretrained in the triangular room still had to learn to use sense cues when they were transferred to the rectangular room; perhaps separating these two aspects of landmark use (the "value" of the landmark itself and its relation to other features of the environment) during pretraining actually worked against the children in the experimental group when they were transferred. Distinguishing between these theoretical possibilities - the role of overshadowing, the relative salience of landmarks, and the characteristics that are functional for a landmark-is an area for future research.

Participants from Learmonth et al.'s (2002) study successfully used a colored wall as a landmark in a large room. However, this ability did not transfer to the small room when these very same 
participants were presented with the same task. In contrast, the children in our study were able to transfer the ability to use the landmark from one situation to the next. What could have led to this difference? Although surface features may have changed, all of the children who participated in the present study practiced in small rooms with approximately the same area. As hypothesized by Learmonth et al. (2002), it may be that there is something about a small room that is at least initially unique. For example, Newcombe (2005) recently hypothesized that it may be difficult to engage spatial orientation mechanisms when it is difficult to move around (as in a small room). A second possibility is that landmarks are more likely to be used when they are far from an observer than when they are close. When an organism moves, there is a larger variability in the locations of proximal features than there is for distal features, making distal landmarks more reliable for reorientation than proximal ones (Newcombe \& Ratliff, 2007). Nevertheless, it did not take very long for the children in our study to become accustomed to the small space; we presume that had Learmonth et al. (2002) continued testing their children in the small space, they would have found results similar to ours.

It has recently been proposed that spatial reorientation may be accomplished by a two-step process (Lee, Shusterman, \& Spelke, 2006). In particular, Lee et al. argue that the first step of reorientation is accomplished through a modular, geometric-based process, whereas the role of landmarks is in the second step, when the landmark's features become directly associated to the goal location. In their study with 4-year-old children, one red and two blue containers were placed in an equilateral triangle configuration. When the hidden object was in the red container, the children readily found it, but when the object was hidden in one of the blue containers, they divided their search equally between the two blue containers. Because the red container could have been used to disambiguate the hiding location of the two blue containers, but was not, the authors concluded that the red container was used as a beacon and not as a landmark.

However, because the children were tested in a circular room, there were really no geometric features that could be associated to the landmark container. Indeed, the children were essentially asked to learn the relations among three discrete objects, which may be a fundamentally different task than the standard reorientation task. Moreover, the children may not have encoded the containers as landmarks because the containers were small and portable, and the children saw them move during the experiment. Objects with these characteristics do not serve as trustworthy landmarks in a more naturalistic setting. In contrast, in our experiment, the colored wall was a permanent landmark in a relatively large, stable structure. Thus, a fairer test of the two step-process proposed by Lee et al. (2006) would be to replicate either our first experiment with the pretraining group or the yellow-wall group of the second experiment and have some of the children search for the hidden object at corners that were not adjacent to the feature wall. If the children fail at this task, then it is possible that the reorientation process occurs by associating the landmark's features to a particular location, as outlined by Lee et al. However, if children succeed when the hidden object is not adjacent to the feature wall, it suggests that the reorientation task is nonmodular and that geometric and landmark information can be adaptively combined (Newcombe, 2005).
At this point, we believe that the results of a study by Newcombe, Jones, and Shallcross (2007) make it likely that the processes underlying young children's reorientation are not modular. The search space they used was an octagon with alternating long and short walls so that there were four geometrically correct and four geometrically incorrect corners. When there was no feature wall, 2- to 3-year-olds could use the shape of the octagon to select a geometrically correct corner. When a red feature wall was added, 3 - to 5-year-olds were additionally able to use the landmark to focus on the hiding location, even when the target corners were not adjacent to the feature wall. Thus, similar to the present findings, landmarks that are a stable part of the environment can be involved in a nonmodular fashion during reorientation.

In a recent review of the spatial orientation literature, $\mathrm{K}$. Cheng and Newcombe (2005) noted that (a) all vertebrate species tested thus far used geometry to respond when continuous surfaces form the geometric properties, (b) all species tested used feature information under some circumstances, and (c) all mammals made systematic rotational (geometric) errors under some circumstances, so all mammals occasionally fail to use feature information. They went on to propose three possible process models to account for the way in which geometric and featural information are used to orient, and to reorient, in any environment. In two of these models geometric information is presumed to be represented modularly (Fodor, 1983); in the third, both types of information are combined in the same representation.

Our data support the third type of model. This model assumes that there is no reason to believe that geometric information is encapsulated; rather, there is much support for the idea that geometric and landmark information are combined (and bound to) the same representation of space. Indeed, once processed at input, this model assumes that geometric and featural information are always put together in the same representation. The representation can be used for spatial orientation and reorientation, and in principle, for other tasks as well. Newcombe (2005) and Newcombe and Ratliff (2007) have called this type of process "adaptive combination" (see also Newcombe \& Huttenlocher, 2006), and our data support the idea that many types of information are combined into a single representation. In real-world navigation, all kinds of information are used to orient and to reorient if needed, including geometry, landmarks, proximal and distal cues, and so on (Newcombe \& Huttenlocher, 2006). Although these different types of information might have different developmental trajectories, from a functional standpoint it makes sense that they should bind to the same representation. A child's learning history would naturally affect the weighting given to different sorts of information; indeed, from an adaptive combination viewpoint it is natural to suppose that experience will influence the weightings in some sort of integrative process. The current data do not allow us to address what factors may determine how and whether different types of experiences influence the weighting of different sorts of information; however, the possibilities have theoretical consequences that are worthy of further investigation.

Because the children in this study learned to combine geometric and landmark cues in about four trials (at most), it seems likely that their abilities to attend, encode, remember, and successfully use landmark features are not being learned "from scratch." Similarly, because K. Cheng's (1986) rats learned to use landmark features in 
a reference memory task in about 30 trials, they, too, were unlikely to have been learning the constellation of cognitive functions underlying this behavior from scratch. Rather, the ability to use landmarks and combine them with geometric cues must be extant in the organism(s), needing only a little practice to become functional. It would benefit the organism to have as many representations of a food location as possible to augment the chances of finding the location at a future date. That this is so should not be surprising; from an ecological standpoint, the type of landmarks that are functional for navigation or used to signal a food supply would rarely change locations with respect to the geometry of the environment.

Our results add to evidence suggesting that children as young as 4 years are fully capable of combining featural and geometric information. They do so even in a small room, within a relatively few trials. Nevertheless, our results are also consistent with the notion that for young children, the use of features is more fragile than, or at least not as automatic as, the use of geometry. Using geometry to reorient appears to occur immediately in either small or large spaces, whereas use of features needs to be catalyzed by experience.

\section{References}

Cheng, K. (1986). A purely geometric module in the rat's spatial representation. Cognition, 23, 149-178.

Cheng, K., \& Gallistel, C. R. (1984). Testing the geometric power of a spatial representation. In H. L. Roitblat, H. S. Terrace, \& T. G. Bever (Eds.), Animal cognition (pp. 409-423). Hillsdale, NJ: Erlbaum.

Cheng, K., \& Newcombe, N. (2005). Is there a geometric module for spatial orientation? Squaring theory and evidence. Psychonomic Bulletin \& Review, 12, 1-23.

Cheng, T. (Producer), Soria, M. (Producer/Director), \& Darnell, E. (Director). (2005). Madagascar [Motion picture]. United States: DreamWorks Animation SKG.

Fodor, J. A. (1983). The modularity of mind. Cambridge, MA: MIT Press. Gouteux, S., \& Spelke, E. (2001). Children's use of geometry and landmarks to reorient in an open space. Cognition, 81, 119-148.

Gouteux, S., Thinus-Blanc, C., \& Vauclair, J. (2001). Rhesus monkeys use geometric and nongeometric information during a reorientation task. Journal of Experimental Psychology: General, 130, 505-519.

Hermer, L., \& Spelke, E. (1994, July). A geometric process for spatial representation in young children. Nature, 370, 57-59.

Hermer, L., \& Spelke, E. (1996). Modularity and development: The case of spatial reorientation. Cognition, 61, 195-232.

Hermer-Vazquez, L., Moffet, A., \& Munkholm, P. (2001). Language, space, and the development of cognitive flexibility in humans: The case of two spatial memory tasks. Cognition, 79, 263-299.
Kelly, D. M., Spetch, M. L., \& Heth, C. D. (1998). Pigeons' encoding of geometric and featural properties of a spatial environment. Journal of Comparative Psychology, 112, 259-269.

Learmonth, A. E., Nadel, L., \& Newcombe, N. S. (2002). Children's use of landmarks: Implications for modularity theory. Psychological Science, $13,337-341$.

Learmonth, A. E., Newcombe, N. S., \& Huttenlocher, J. (2001). Toddlers' use of metric information and landmarks to reorient. Journal of Experimental Child Psychology, 80, 225-244.

Lee, S. A., Shusterman, A., \& Spelke, E. S. (2006). Reorientation and landmark-guided search by young children. Psychological Science, 17, $577-582$.

Newcombe, N. S. (2005). Evidence for and against a geometric module: The roles of language and action. In J. Rieser, J. Lockman, \& C. A. Nelson (Eds.), Minnesota Symposia on Child Psychology: Vol. 33. Action as an organizer of learning and development (pp. 221-241). Mahwah, NJ: Erlbaum.

Newcombe, N. S., \& Huttenlocher, J. (2006). Development of spatial cognition. In W. Damon \& R. Lerner (Series Eds.) \& D. Kuhn \& R. Siegler (Vol. Eds.), Handbook of Child Psychology: Vol. 2. Perception, Cognition, \& Language (6th ed.). New York: Wiley.

Newcombe, N. S., Jones, M., \& Shallcross, W. L. (2007, March). How are geometric and featural information used to reorient in a complex space? In S. F. Lourenco (Chair), Spatial representation in young children: How is geometric and non-geometric location information processed? Symposium conducted at the meeting of the Society for Research in Child Development, Boston.

Newcombe, N. S., \& Ratliff, K. R. (2007). Explaining the development of spatial reorientation: Modularity-plus-language versus the emergence of adaptive combination. In J. Plumert \& J. Spencer (Eds.), The emerging spatial mind (pp.53-76). New York: Oxford University Press.

Sovrano, V. A., Bisazza, A., \& Vallortigara, G. (2002). Modularity and spatial reorientation in a simple mind: Encoding of geometric and nongeometric properties of a spatial environment by fish. Cognition, 85 , B51-B59.

Vallortigara, G., Zanforlin, M., \& Pasti, G. (1990). Geometric modules in animals' spatial representations: A test with chicks (Gallus gallus domesticus). Journal of Comparative Psychology, 104, 248-254.

Wall, P. L., Botly, L. C. P., Black, C. K., \& Shettleworth, S. J. (2004). The geometric module in the rat: Independence of shape and feature learning in a food finding task. Learning \& Behavior, 32, 289-298.

Wang, R. F., Hermer, L., \& Spelke, E. (1999). Mechanisms of reorientation and object localization by children: A comparison with rats. Behavioral Neuroscience, 113, 475-485.

Received August 30, 2006

Revision received June 6, 2007

Accepted June 18, 2007 